\title{
Survivin is essential for fertile egg production and female fertility in mice
}

\author{
Z-Z Jiang ${ }^{1,2,8}$, M-W Hu ${ }^{1,2,8}$, Z-B Wang ${ }^{1,8}$, L Huang ${ }^{1,2}$, F Lin ${ }^{1}$, S-T Qi ${ }^{1,2}$, Y-C Ouyang ${ }^{1}$, H-Y Fan ${ }^{3}$, H Schatten ${ }^{4}$, TW Mak ${ }^{5,6,7}$ and Q-Y Sun ${ }^{\star, 1}$
}

Survivin is the smallest member of the inhibitor of apoptosis protein (IAP) family and acts as a bifunctional protein involved in mitosis regulation and apoptosis inhibition. To identify the physiological role of Survivin in female reproduction, we selectively disrupted Survivin expression in oocytes and granulosa cells (GCs), two major cell types in the ovary, by two different Cre-Loxp conditional knockout systems, and found that both led to defective female fertility. Survivin deletion in oocytes did not affect oocyte growth, viability and ovulation, but caused tetraploid egg production and thus female infertility. Further exploration revealed that Survivin was essential for regulating proper meiotic spindle organization, spindle assembly checkpoint activity, timely metaphase-to-anaphase transition and cytokinesis. Mutant mice with Survivin depleted in GCs showed reduced ovulation and subfertility, caused by defective follicular growth, increased follicular atresia and impaired luteinization. These findings suggest that Survivin has an important role in regulating folliculogenesis and oogenesis in the adult mouse ovary.

Cell Death and Disease (2014) 5, e1154; doi:10.1038/cddis.2014.126; published online 27 March 2014

Subject Category: Experimental Medicine

In mammals, ovarian folliculogenesis is a process regulated by complex networks composed of various endocrine, paracrine and autocrine factors that interact coordinately. ${ }^{1}$ A follicle is composed of three distinct cell types: an oocyte and two different kinds of somatic cells, granulosa cells (GCs) and theca cells. The oocyte is continuously arrested at the germinal vesicle (GV) stage during follicle development, and the fully grown oocyte in the mature follicle begins the maturation process upon stimulation by the gonadotropin surge, as indicated by a significant event: GV breakdown (GVBD), followed by spindle organization and chromosome alignment as an important process for correct chromosome segregation. Following the small first polar body extrusion (PBE) produced by unequal cytokinesis the mature eggs are now ready for fertilization. ${ }^{2,3}$

Successful oogenesis and oocyte maturation rely on close cooperation and communication between the oocyte and surrounding GCs. Receptors expressed on GCs such as the FSH receptor (Fshr) and LH receptor (Lhcgr), and factors secreted from GCs such as estradiol and insulin-like growth factor (IGF) are proved to be essential for folliculogenesis. ${ }^{4}$ Moreover, apoptosis of GCs was observed to occur earlier than that of oocytes and theca cells in atretic follicles, suggesting their vital role in folliculogenesis and follicle atresia. $^{4-6}$
Survivin, the smallest member of the inhibitors of apoptosis protein (IAPs) family, is a $16.5-\mathrm{kDa}$ protein and contains a single repeat of BIR domain that is essential for the caspaseinhibitory function. ${ }^{7}$ After years of research, Survivin has been shown to have multiple roles that are not limited to apoptosis inhibition but also to regulation of the mitotic spindle checkpoint. On one hand, Survivin is proved to be a caspase inhibitor given its BIR domain, which is common to the other IAP family members. Studies showed that Survivin, associated with the hepatitis $B$ X-interacting protein (HBXIP) or X-linked IAP (XIAP), inhibited activation of Caspase-9. ${ }^{8}$ Survivin also acts as a downstream target protein involved in several important signaling pathways, the PI3K/Akt pathway, mTOR pathway, ERK pathway, tumour suppressors (p53, PTEN) and oncogenic (Ras, Bcl-2) signaling pathways. ${ }^{9}$ On the other hand, Survivin together with INCENP, Borealin and Aurora B form the chromosomal passenger complex (CPC) for regulation of cell division. ${ }^{10,11}$ During mitosis, Survivin locates to centromeres during prophase/metaphase, relocates to the spindle mid-zone during anaphase/telophase and disappears at the end of telophase. ${ }^{12}$ Survivin is ubiquitously expressed during embryonic and fetal development and has been proved to have roles in both neurogenesis and hematopoiesis; ${ }^{13,14}$ it is also expressed in various cancers.

\footnotetext{
${ }^{1}$ State Key Laboratory of Reproductive Biology, Institute of Zoology, Chinese Academy of Sciences, Beijing 100101, China; ${ }^{2}$ University of Chinese Academy of Sciences, Beijing 100101, China; ${ }^{3}$ Life Science Institute, Zhejiang University, Hangzhou 310000 , China; ${ }^{4}$ Department of Veterinary Pathobiology, University of Missouri, Columbia, MO 65211, USA; ${ }^{5}$ Advanced Medical Discovery Institute, Ontario Cancer Institute, University of Toronto, Toronto, Ontario, Canada M5G 2C1; ${ }^{6}$ Department of Medical Biophysics, University of Toronto, Toronto, Ontario, Canada M5G 2C1 and ${ }^{7}$ Department of Immunology, University of Toronto, Toronto, Ontario, Canada M5G 2C1 ${ }^{*}$ Corresponding author: Q-Y Sun, State Key Laboratory of Reproductive Biology, Institute of Zoology, Chinese Academy of Sciences, \#1 Beichen West Road, Chaoyang, Beijing 100101, China. Tel/Fax: +86 106480 7050; E-mail: sunqy@ioz.ac.cn

${ }^{8}$ These authors contributed equally to this work and should be regarded as joint first authors.

Keywords: folliculogenesis; apoptosis; granulosa cell; meiosis; oocyte; Survivin

Abbreviations: GCs, granulosa cells; GVBD, germinal vesicle breakdown; SAC, spindle assembly checkpoint; APC/C, anaphase-promoting complex/cyclosome; PBE, polar body extrusion; MCC, mitotic checkpoint complex; COC, cumulus cell-oocyte complex; CL, corpora lutea; CC3, cleaved caspase 3; TUNEL, terminal deoxynucleotide transferase-mediated deoxyuridine triphosphate nick end labeling

Received 09.1.14; revised 26.2.14; accepted 27.2.14; Edited by A Stephanou
} 
In this study, we generated mutant mice with specific deletion of Survivin in oocytes and GCs to investigate the function of Survivin within ovarian follicles in vivo. For the first time, we report here that Survivin is expressed in both mouse oocytes and GCs and it is essential for female fertility. Oocytespecific deletion of Survivin did not affect oocyte survival and ovulation, but led to female infertility due to tetraploid eggs derived from failure of cytokinesis and first polar body emission. Survivin mutation in oocytes also caused misorganized spindles and a dysfunctional spindle assembly checkpoint (SAC). In addition, deletion of Survivin in GCs caused compromised follicular development, reduced ovulation and impaired luteinization, which led to defective ovarian function and female subfertility.

\section{Results}

Oocyte-specific/GC-specific disruption of the Survivin gene. The mutant mice with oocyte-specific and GC-specific disruption of the Survivin gene, in which exon II of the Survivin gene was respectively targeted in oocytes and GCs, ${ }^{15}$ were generated by crossing Sur ${ }^{F / F}$ mice with transgenic mice expressing Gdf9 or Cyp19 promotermediated Cre recombinase. Survivin always expressed in oocytes and GCs in the Sur F/F ovary (Figure 1a). Western blot analysis showed in Sur F/F; Gdf9-Cre mouse oocytes and Sur $^{F / F}$; Cyp19-Cre mouse GCs, the Survivin protein was depleted (Figure 1b). Immunofluorescent analysis of oocytes from Sur F/F; Gdf9-Cre female revealed loss of Survivin localization on kinetochores (Figure 1c).

Survivin is essential for female fertility. Breeding assays showed that female Sur ${ }^{F / F}$; Gdf9-Cre mice were completely infertile, while the Sur ${ }^{F / F}$; Cyp19-Cre mice were evidently subfertile and gave birth to about $70 \%$ fewer pups than control mice (Figure 1d). The decreased number of pups born in Sur ${ }^{F / F}$; Cyp19-Cre mice appeared to be related to decreased ovulation as seen in the result of the superovulation assay (Figure 1e).

Depletion of Survivin causes spindle defects, chromosome misalignment, premature anaphase entry and first PBE failure of oocytes. To investigate the primary defect leading to female infertility after oocyte-specific Survivin depletion, we first performed superovulation. We found that oocytes were ovulated normally, while no polar body was extruded in Sur ${ }^{F / F}$; Gdf9-Cre mice (Figure 2a). To further understand the defects in oocytes caused by Survivin deletion, we employed immunofluorescent staining to observe spindles and chromosomes in the oocytes. It was observed that a number of misaligned chromosomes existed in superovulated Sur F/F; Gdf9-Cre oocytes (see white arrows in Figure 2b), and the rate of oocytes with misaligned chromosomes was significantly higher than that in Sur F/F $^{2}$ eggs $(92.3 \pm 5.1 \%$ versus $13.3 \pm 8.9 \%$ ) (Figure $2 \mathrm{c})$. Another interesting phenotype of Sur ${ }^{F / F}$; Gdf9-Cre oocytes is that they displayed defective spindle morphologies (see red arrows in Figure 2b). In many abnormal spindles, bundles of microtubules were seen scattered with chromosomes attached to them (see arrows in Figure 2b'). In addition, we performed chromosome spreads to address chromosome morphology. As expected, Sur F/F; Gdf9-Cre oocytes displayed 40 univalent chromosomes that were twice in number compared with those in Sur ${ }^{F / F}$ oocytes (Figure 2d), indicating that oocytes yielded from female Sur ${ }^{F / F}$; Gdf9-Cre mice all displayed tetraploidy. Ovaries of indicated genotypes were fixed after injection of PMSG (48h) and hCG (8h). After H\&E staining, we found that oocytes in follicles remained at the $\mathrm{MI}$ stage in the Sur ${ }^{F / F}$ group, whereas chromosomes were segregating (Figure 2e, white arrows), or an abnormal polar body was extruding (Figure 2e, black arrow) in Sur ${ }^{F / F}$; Gdf9Cre oocytes, indicating premature anaphase entry.

The above phenotypes, especially the precocious anaphase entry, were further confirmed by incubating $S u r^{F / F}$; Gdf9-Cre GV oocytes in M2 medium in vitro. After culture for $2 \mathrm{~h}$, the oocytes that had undergone GVBD were counted and the results showed no obvious difference in GVBD rates between Sur $^{F / F}$ and Sur F/F; Gdf9-Cre oocytes $(88.4 \pm 2.0 \%$ versus $83.3 \pm 0.9 \%$ ) (Supplementary Figure S1A). As was the case in in vivo experiments, we observed a significantly lower PBE rate in Sur F/F; Gdf9-Cre oocytes $(2.9 \pm 3.9 \%$ versus $75.9 \pm 0.7 \%$ ) (Supplementary Figure S1B) after culture for $12 \mathrm{~h}$. It was observed that multipolar spindles and misaligned chromosomes dominantly existed in Sur ${ }^{F / F}$; Gdf9-Cre oocytes after culture (Supplementary Figure S1C). There was a significant increase in frequency of abnormal bipolar spindles $(82.1 \pm 6.2 \%$ versus $14.2 \pm 7.2 \%$, Supplementary Figure S1D) and misaligned chromosomes $(97.4 \pm 3.4 \%$ versus $20.1 \pm 3.2 \%$, Supplementary Figure S1E). In Sur ${ }^{F / F}$; Gdf9Cre oocytes, abnormal spindles contained more than two poles and scattered poles with different orientations, which was confirmed by staining of $\gamma$-tubulin (see arrows in Supplementary Figure S1F). To address whether homologous chromosome segregation prematurely took place in Sur'F/F; Gdf9-Cre oocytes, we performed chromosome spreading and found prematurely segregated homologs after culture for $8 \mathrm{~h}$ (Supplementary Figure S1G, left). Univalent chromosomes were found in oocytes from both mutant genotypes after culture for $12 \mathrm{~h}$ (Supplementary Figure S1G, right), suggesting that homologous chromosomes were precociously segregated in Sur/F; Gdf9-Cre oocytes.

Depletion of Survivin led to failure of spindle checkpoint protein localization to kinetochores, and premature activation of anaphase-promoting complex/cyclosome. Considering the phenotypes of chromosome misalignment and precocious chromosome segregation, we next asked whether the SAC is under proper control in Sur F/F; Gdf9-Cre oocytes. In Sur F/F oocytes, there was clear localization of Bub3 and BubR1 (two SAC proteins) ${ }^{16,17}$ at kinetochores. In sharp contrast, there was no visual centromeric localization of both SAC proteins in Sur/F; Gdf9-Cre oocytes (Figures 3a and b), suggesting that Survivin was required for the localization of SAC proteins to kinetochores. Bub3, BubR1, Mad2 and Cdc20 form the mitotic checkpoint complex (MCC) that inhibits the ability of Cdc20 to activate the anaphasepromoting complex/cyclosome (APC/C) in mitosis ${ }^{18}$ and a similar mechanism was determined for meiosis. ${ }^{19}$ For the evaluation of premature activation of $\mathrm{APC} / \mathrm{Ccdc} 20$, we used time-lapse live imaging to observe the degradation of Cyclin 
a

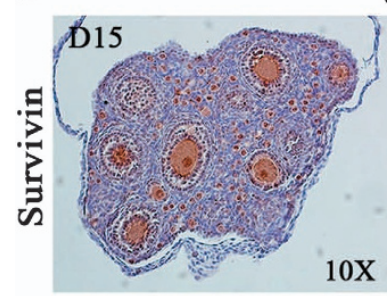

WT

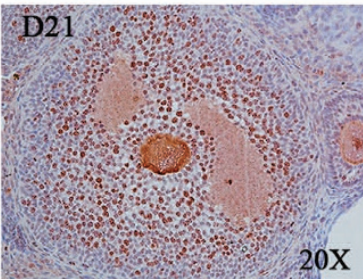

b

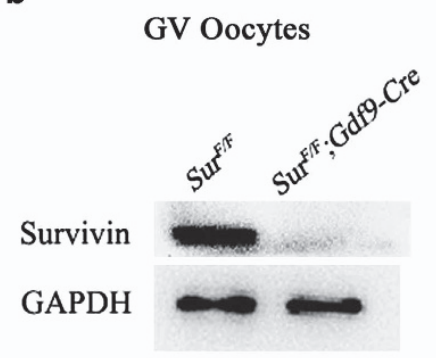

C

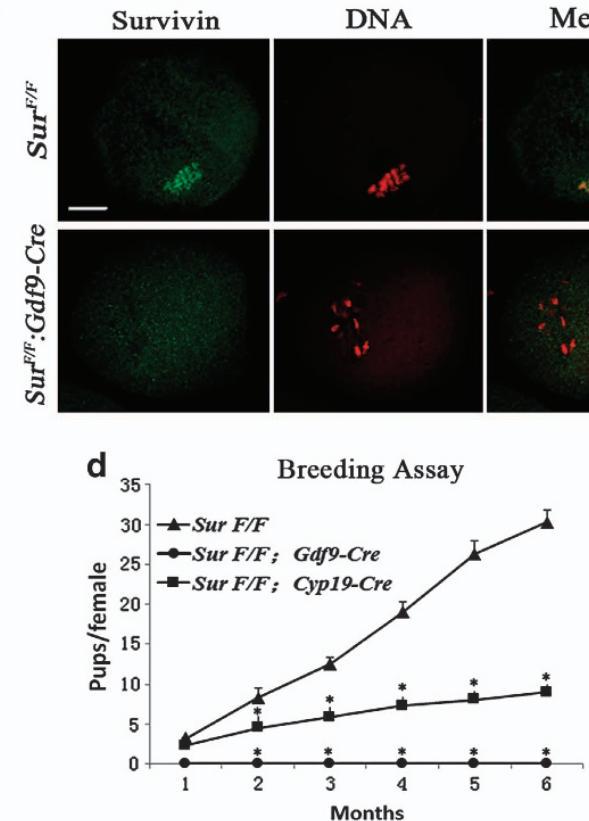

Merge

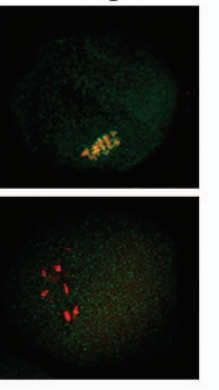

e

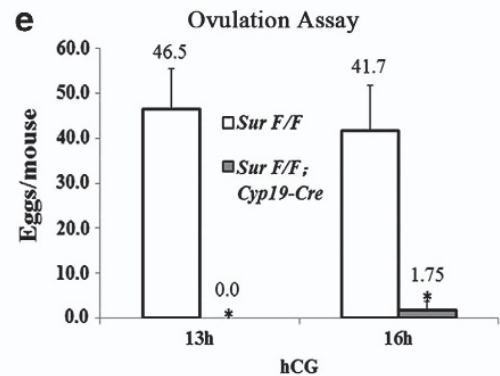

Figure 1 Survivin expression in oocytes and granulosa cells is essential for female fertility. (a) Expression of Survivin protein in ovaries of WT D15 and D21 mice. (b) Verification of Survivin deletion in oocytes and granulosa cells from mice with indicated genotypes. GV oocytes were collected from minced ovaries and 200 oocytes were used for each sample. GAPDH was used as a loading control. (c) Localization of Survivin (green) in Sur ${ }^{F / F}$; Gdfg-Cre and SuF ${ }^{F / F}$ oocytes. Oocytes at $8 \mathrm{~h}$ of culture were fixed for immunofluorescent staining. Red, DNA. Bar $=20 \mu \mathrm{m}$. (d) Infertility of the female Sur ${ }^{F / F} ;$ Gdfg-Cre mice and subfertility of the female Sur ${ }^{F / F}$; Cyp19-Cre mice. Continuous breeding assessment showed the cumulative number of progeny per female mouse. (e) Lack of or defective ovulation in Sur F/F; Cyp19-Cre mice. Oocytes were collected at 13 and $16 \mathrm{~h}$ of $\mathrm{hCG}$ treatment, respectively ( $n=6$ for each genotype in each group). ${ }^{*} P<0.05$

B1 which is one of the key substrates of APC/Ccdc20. As shown in the movies, we found that Cyclin B1 started to be degraded in Sur F/F; Gdf9-Cre oocytes at about $2 \mathrm{~h}$ ahead of that in $S u r^{F / F}$ oocytes (Figures $3 c$ and $d$, also see Supplementary Movie $1 \mathrm{~A}$ and $\mathrm{B}$ in Supplementary Material), which was confirmed by the quantitation of total fluorescence of Cyclin B1-GFP in both the genotypes (Figure $3 e$ ). This result validated the finding that deletion of Survivin resulted in failure of SAC assembly to kinetochores for inhibition of Cdc20, which triggers precocious anaphase I onset during the first meiosis.

Depletion of Survivin caused cytokinetic abscission failure. To explore the underlying reason for PBE failure, we performed live oocyte imaging to analyze spindle dynamics (Figure 4) and F-actin (Figure 5) during oocyte maturation. In Sur/F; Gdf9-Cre oocytes, it was shown that the midbody microtubules were absent at telophase I (red arrow, Figure 4b, also see Supplementary Movie 2B in Supplementary Material) when cytokinesis was initiated, which led to unequal chromosome segregation (white arrows, Figures $4 \mathrm{~b}$ and $5 \mathrm{~b}$, also see Supplementary Movies 2A and 3B in Supplementary Material). In addition, the rate of transient PBE (membrane protrusion for accuracy) at indicated time points showed that the transient PBE rate of Survivin-deficient oocytes reached the peak at $8 \mathrm{~h}$ of culture and all the extruding polar bodies were abnormal, being smaller than the normal size (red and white arrows, Figure $4 \mathrm{c}$ ), and further retracted within $2 \mathrm{~h}$ (Figure $4 \mathrm{~d}$, $10 \mathrm{~h})$. In $\mathrm{Sur}^{\mathrm{F} / \mathrm{F}}$ oocytes, the contractile ring was present throughout the PBE process (red arrows, Figure 5a, also see Supplementary Movie $3 A$ in Supplementary Material). Whereas in $\mathrm{Sur}^{\mathrm{F} / \mathrm{F}}$; Gdf9-Cre oocytes, the assembly of $\mathrm{F}$-actin at the midbody (contractile ring) was not disturbed but disappeared soon along with the retraction of the polar body (red arrows, Figure 5b, also see Supplementary Movie 3B in Supplementary Material), suggesting abscission failure. Immunofluorescent staining showed complete absence of the midbody spindle at telophase in $S u r^{F / F}$; Gdf9-Cre oocytes (Figure 5c). In meiosis, it has been reported that Aurora $C$ instead of Aurora $B$ has pivotal roles in oocyte maturation ${ }^{20,21}$ and Aurora $B$ is responsible for 
a

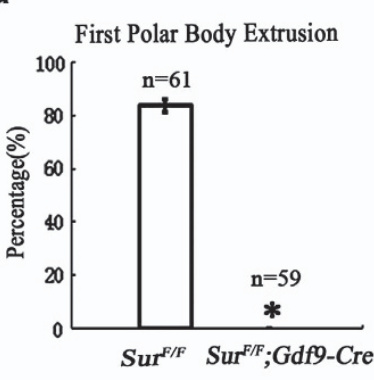

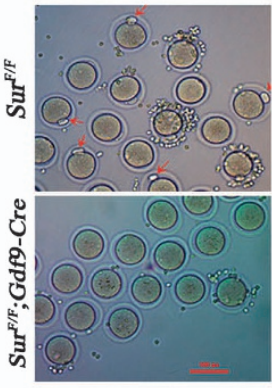

b

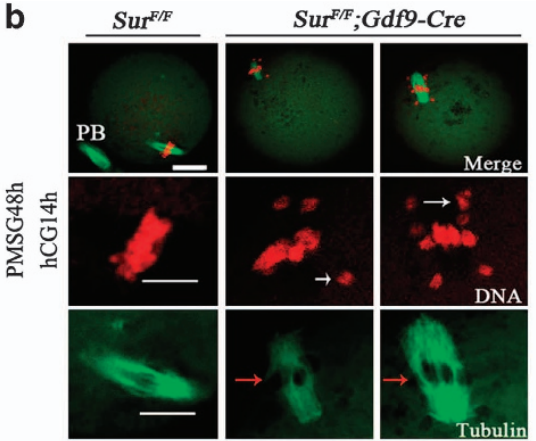

C Misaligned Chromosome

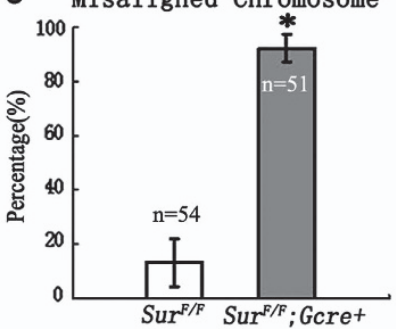

e

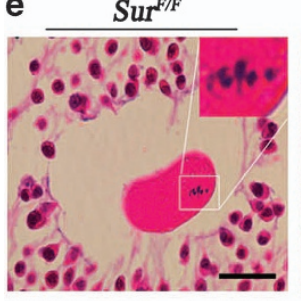

d
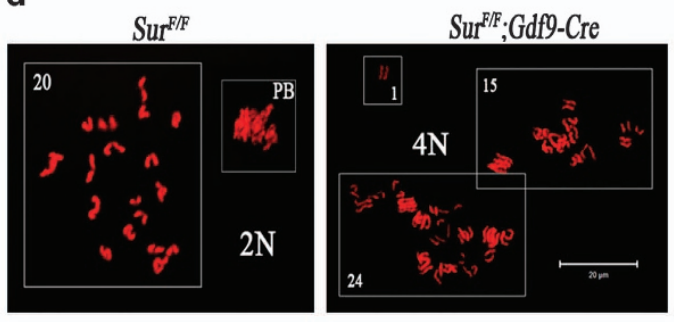

PMSG: 48h hCG: 8h

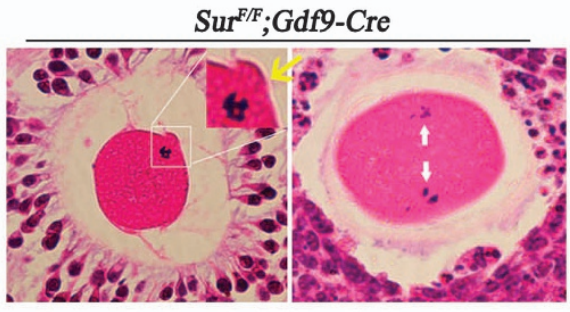

b'

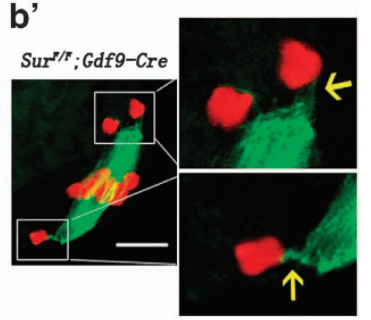

Figure 2 Deletion of Survivin disturbed oocyte maturation in vivo. (a) Percentages of eggs with first polar body extrusion from superovulated Sur/F and Sur/F; Gdf9-Cre females, respectively, after injection of PMSG (48 h) and hCG (12-14 h). Red arrows, first polar body. Bar $=100 \mu \mathrm{m} .{ }^{*} P<0.0001$. (b and b') Immunofluorescent staining of superovulated eggs. White arrows showed misaligned chromosomes and red arrows showed abnormal spindle poles in (b). Yellow arrows showed bundles of microtubules in (b'). Red, DNA; Green, $\alpha$-tubulin; PB, polar body. Scale bars: $20 \mu \mathrm{m}$ in (b) (Merge); $10 \mu \mathrm{m}$ in (b) (DNA and Tubulin) and (b'). (c) Rates of oocytes with misaligned chromosomes in superovulated Sur/F and Sur ${ }^{F / F}$; Gdfg-Cre oocytes, respectively. ${ }^{*} P<0.0001$. (d) Chromosome spreads of superovulated oocytes from the indicated genotypes. Polar body (PB) and the numbers of chromosomes were indicated. Bar $=20 \mu \mathrm{m}$. (e) Hematoxylin and eosin-stained ovary sections from Sur ${ }^{F / F}$ and Sur $\mathrm{F}^{F / F}$; GdfgCre females. Ovaries of the indicated genotypes were fixed after injection of PMSG $(48 \mathrm{~h})$ and $\mathrm{hCG}(8 \mathrm{~h})$. Only oocytes and cumulus cells of the sections were shown. Yellow arrow showed membrane protrusion and white arrows showed the segregating chromosomes

abscission in mitosis. ${ }^{22}$ Here, we found that the assembly of Aurora C (Myc-conjugated) in the contractile ring was disturbed in Sur ${ }^{/ / F}$; Gdf9-Cre oocytes (Figure 5d). Taken together, as shown in Figure 5e, oocyte-specific deletion of Survivin impaired the assembly and maintenance of midbody microtubules that ensures proper chromosome segregation, and caused cytokinetic abscission failure, followed by retraction of the extruding polar body.

Disruption of Survivin in GCs causes defects in folliculogenesis, ovulation and luteinization. Since the Sur F/F; Cyp19-Cre mice could barely ovulate oocytes in the superovulation assay, we then examined whether folliculogenesis was defective. At $24 \mathrm{~h}$ of PMSG treatment, the secondary follicles were growing fast, filled with rapidly increasing GCs in control mice; in contrast, unhealthy follicles with sparsely distributed GCs were observed in Sur $^{F / F}$; Cyp19-Cre mice (Supplementary Figure S2A). This tendency became more obvious after $48 \mathrm{~h}$ of PMSG treatment (Figure 6a). The granulosa layers of $\operatorname{Sur}^{F / F}$; Cyp19-Cre mice were much thinner than the control. To quantify this discrepancy, the diameter of antral follicles was measured and a $20 \%$ decrease in mutant mice was found compared with control mice (Supplementary Figure S2B, right panel). This result was also supported by the smaller size and decreased weight of ovaries from SurF/Fyp19-Cre mice (Supplementary Figure S2B, left panel).

Typically in mutant mice, there were only $4-5$ layers of membrana GCs (black arrow) and only one layer of cumulus cells (yellow arrow); furthermore, many of these GCs displayed pyknotic nuclei that indicated morphological apoptosis (Figure 6a, 20X). To test whether the dominant antral follicles could maintain ovulation, we administered hCG at $48 \mathrm{~h}$ of PMSG to simulate ovulation. At $8 \mathrm{~h}$ of $\mathrm{hCG}$ priming, the cumulus cell-oocyte complex (COC) had undergone expansion in control ovaries as expected; however, there were too few cumulus cells (Supplementary Figure S2C, yellow arrow) to expand and even thinner membrana granulosa 

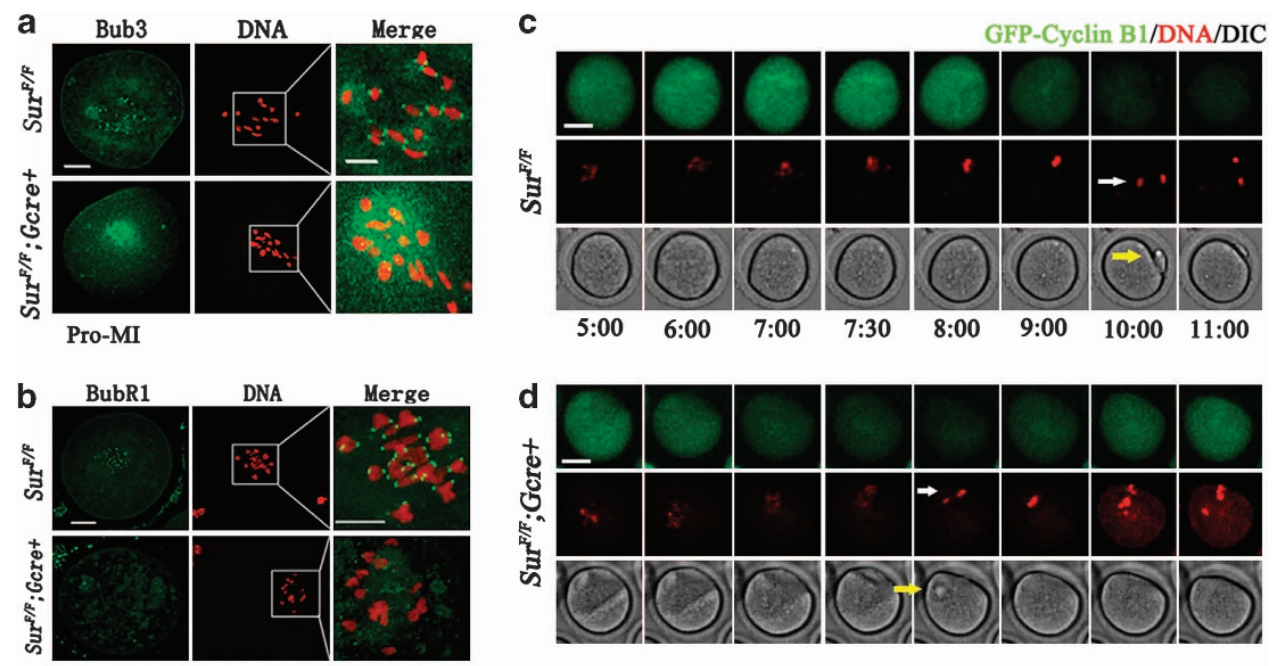
Pro-MI
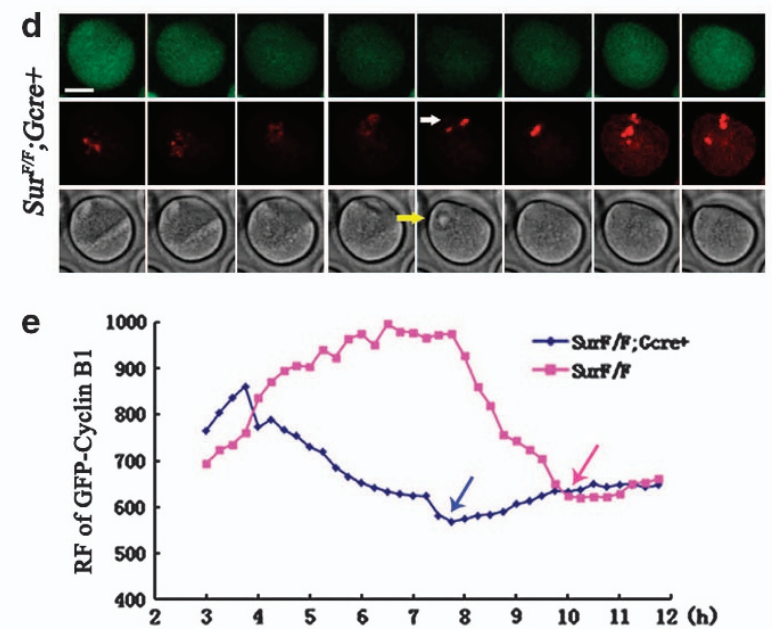

Figure 3 Precocious anaphase initiation during Sur ${ }^{F / F}$; Gdfg-Cre oocyte maturation. (a and $\mathbf{b}$ ) Immunofluorescent staining showing the localization of spindle assembly checkpoint (SAC): proteins Bub3 (a) and BubR1 (b) in oocytes at the Pro-Ml stage of the indicated genotypes. Green: Bub3 in (a), BubR1 in (b); Red, DNA. Scale bars: $20 \mu$ m in (a) (Bub3), (b) (BubR1); $10 \mu \mathrm{m}$ in (a) and (b) (Merge). (c and d) Dynamics of Cyclin B1-GFP fluorescence during Sur ${ }^{F / F}$ and Sur F/F; Gdf9-Cre oocyte maturation. Oocytes microinjected with Cyclin B1-GFP mRNA were transferred to the live imaging station after $2 \mathrm{~h}$ of incubation. Time points indicated the time lapse from the start of corresponding Supplementary Movie $1 \mathrm{~A}$ and B. White arrows showed segregated chromosomes in (a) and (b). Yellow arrow showed polar body in (a) and membrane protrusion in (b). Bar $=25 \mu \mathrm{m}$. (e) Cyclin B1-GFP fluorescence intensities in Sur ${ }^{F / F}$ and Sur ${ }^{F / F}$; Gdf9-Cre oocytes. Time points indicated the time elapsed from the start of corresponding Supplementary Movie $1 \mathrm{~A}$ and B. $\mathrm{RF}$, relative fluorescence. Arrows indicated the time points of polar body extrusion in SuF ${ }^{F / F}$ oocytes and membrane protrusion in Sur ${ }^{F / F}$; Gdf9-Cre 0ocytes

(Supplementary Figure S2C, black arrow) in the preovulatory follicles of mutant ovaries. As the process continued, most COCs of mutant mice failed to ovulate and these follicles were left in the ovaries and turned into atretic follicles (Figure 6b, asterisks).

We further investigated whether the luteinization process was affected at 24 and $48 \mathrm{~h}$ of $\mathrm{hCG}$ administration when the corpora lutea $(\mathrm{CL})$ of the control mice were well developed (Supplementary Figure S2D; Figure 6c, left panel), and found that luteinization was slower in the mutant mice. Many atretic follicles were not filled with luteinized GCs at $24 \mathrm{~h}$ of hCG treatment (Supplementary Figure S2D, right panel, asterisks), and the development of CLs did not complete even after $48 \mathrm{~h}$ of hCG administration (Figure $6 \mathrm{c}$, right panel, asterisks). CL counting also confirmed that the numbers of developed CLs displayed significant differences between the Sur F/F; Cyp19-Cre mice and control mice (Supplementary Figure S2E, right panel). The results from comparison of ovary size and weight between the two groups (Supplementary Figure S2E, left panel) may be further supporting evidence for the conclusion that luteinization of mutant mice was impaired. Analysis of serum estradiol and progesterone showed a marked decrease in both hormone levels in comparison with the control mice (Figure 6d).
Survivin deletion led to GCs apoptosis and impaired ovarian function. To characterize the observed changes in Survivin knockout ovaries at the cellular level, we first examined the proliferative rate of GCs in the developing follicles. On the basis of immunostaining for the mitosis marker phosphohistone $\mathrm{H} 3(\mathrm{pHH})$ (Supplementary Figure $\mathrm{S} 3 \mathrm{~A})$, it appeared that there was no significant difference in GC proliferation especially in normal secondary follicles between the two groups. To test the apoptosis level in mutant ovaries, we next employed the terminal deoxynucleotide transferase-mediated deoxyuridine triphosphate nick end labeling (TUNEL) assay and immunostaining for cleaved caspase 3 (CC3). The numbers of apoptotic follicles (Figure 7a) and CC3-positive GCs (Figure 7b) were significantly increased in the PMSG-primed Survivin mutant ovaries. When the Survivin knockout GCs were isolated and cultured in vitro for immunofluorescent staining, the number of CC3-positive GCs increased even further (Figure 7c). Western blot analysis showed that the levels of pro-caspase 9 and pro-caspase 3 were reduced in the Survivin mutant GCs, indicating more cleavage activity of these caspases (Supplementary Figure S3B). As shown in Figure 7d, the mutant GCs showed an evident decrease in mRNA expression levels of Fshr and Lhgcr. Moreover, we examined the expression of aromatase (Cyp19a1) and found that it was 


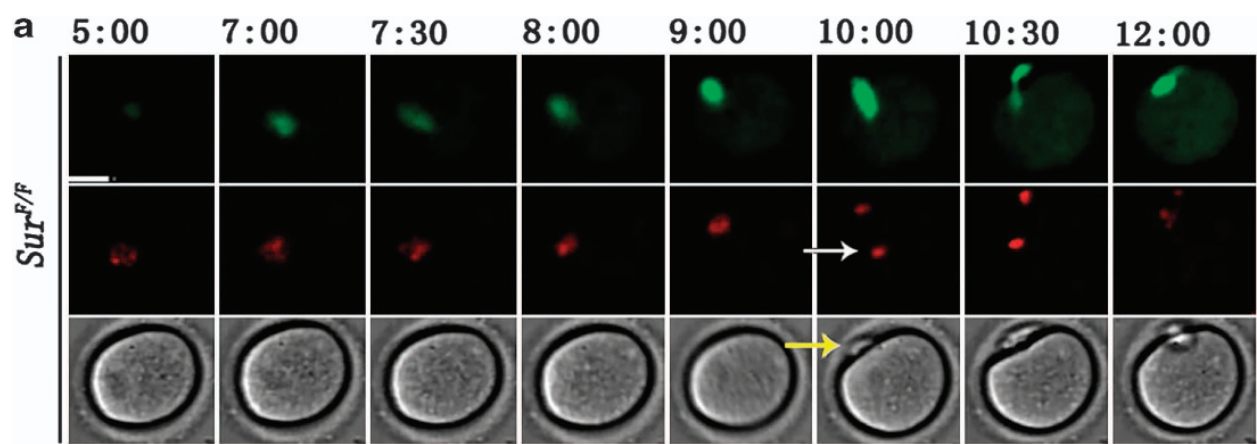

b

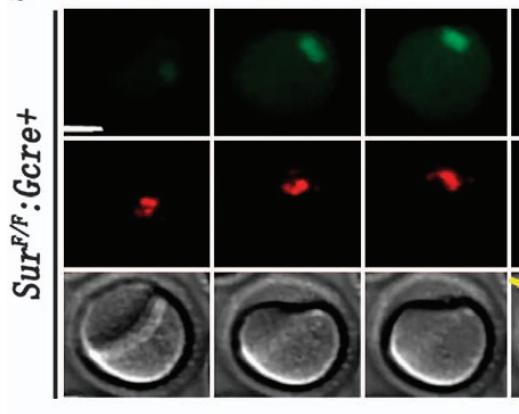

$\beta$-Tubulin-GFP/DNA/DIC
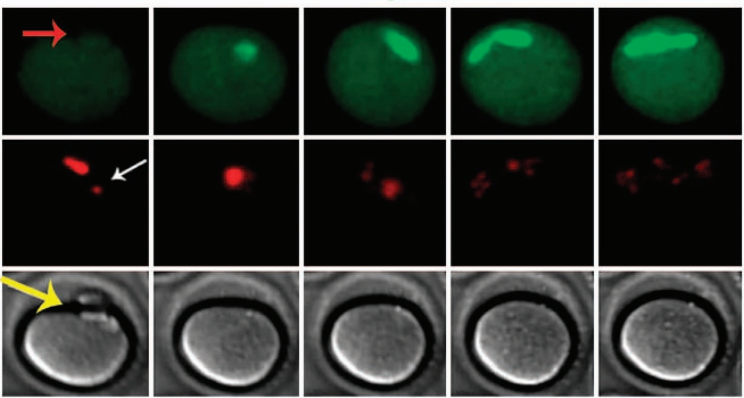

C Polar body and membrane protrusion
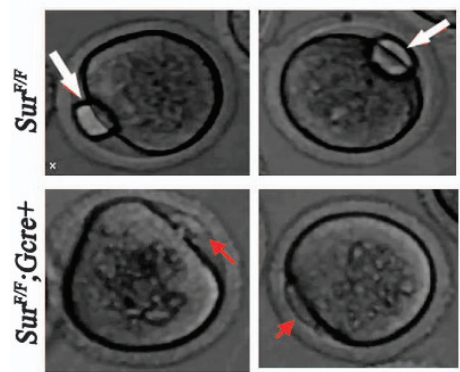

d

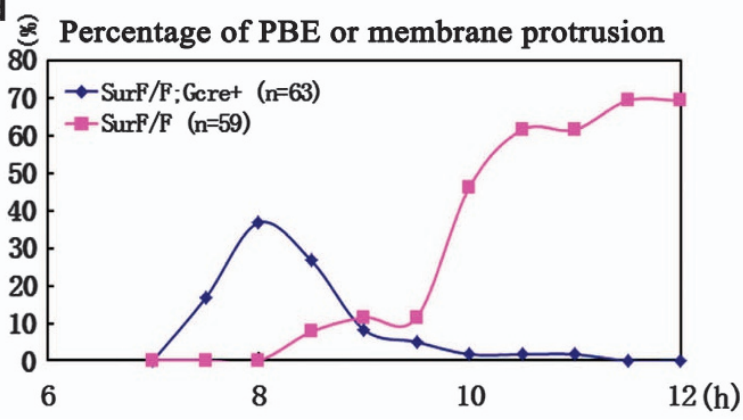

Figure 4 Impaired midbody in Sur ${ }^{F / F}$; Gdf9-Cre oocytes at late meiosis I. (a and b) Dynamics of spindle and chromosomes during maturation in SuF ${ }^{F / F}$ and Sur ${ }^{F / F}$; GdfgCre oocytes. $\beta$-Tubulin-GFP mRNAs were microinjected into GV oocytes of the indicated genotypes and oocytes were transferred to the live imaging station after $2 \mathrm{~h}$ of incubation when most oocytes had undergone GVBD. Time points indicated the time elapsed from the start of corresponding Supplementary Movie 2A and B. Red arrow indicated the absence of spindle midbody microtubules in (b); White arrows indicated segregated chromosomes in (a) and (b); yellow arrow showed the polar body in (a) and membrane protrusions in (b). Bar $=25 \mu \mathrm{m}$. (c) Polar bodies extruded by Sur ${ }^{F / F}$ oocytes at $12 \mathrm{~h}$ of culture (upper row, white arrows) and membrane protrusions in Sur F/F; $^{F}$ GdfgCre oocytes at $8 \mathrm{~h}$ of culture (lower row, red arrows). (d) Cumulative rates of polar body extrusion and membrane protrusion during maturation of Sur ${ }^{\mathrm{F} / F}$ and Sur ${ }^{\mathrm{F} / F}$; Gdf9-Cre oocytes. The numbers of analyzed oocytes were indicated $(n)$

dramatically downregulated in Sur ${ }^{F / F}$; Cyp19-Cre mice, suggesting that the differentiation of GCs to the preovulatory stage was impaired.

\section{Discussion}

We found that Survivin in both oocyte and surrounding GCs is essential for female fertility. During follicle development, primordial follicles with Survivin depletion in the oocyte could develop into antral follicles with normal granulosa and cumulus cells, and release eggs. However, deletion of Survivin in oocytes caused impaired oocyte maturation and infertility. Our findings also provided evidence that specific deletion of Survivin in GCs led to abnormal ovarian function and female subfertility.
Survivin is required for spindle assembly, SAC protein kinetochore localization and the accurate regulation of anaphase onset during oocyte meiotic maturation. In mitosis, cells lacking Survivin are unable to align their chromosomes, fail to recruit Aurora B to kinetochores and become polyploid at a very high frequency. ${ }^{23}$ Survivindepleted cells displayed delayed cell-cycle progression and accumulated in prometaphase with misaligned chromosomes. ${ }^{24}$ In human cells, Survivin also regulates assembly and stability of microtubules during late mitosis. ${ }^{25} \mathrm{We}$ recently showed that Survivin knockdown in vitro led to precocious PBE but did not affect the bipolar spindle formation. ${ }^{26}$ In our previous studies, we showed that BubR1 knockdown induced acceleration of mouse oocyte meiotic progression, ${ }^{17}$ while Bub3 deletion by RNAi did not disturb the meiotic process but abrogated oocyte meiotic 
a
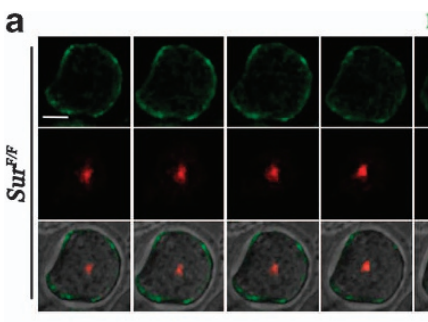

FITC-phalloidin/DN/A/Merge

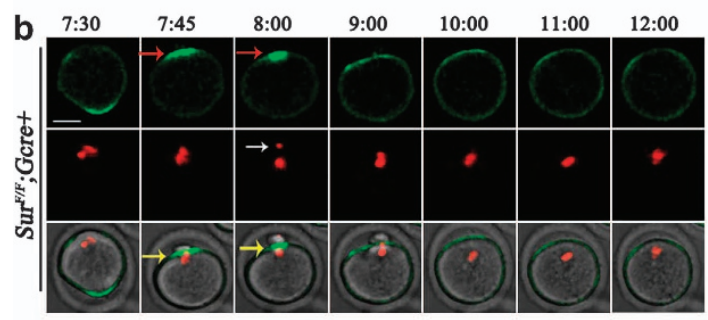

e

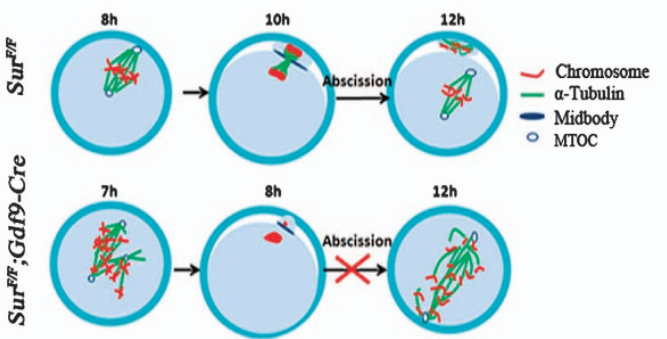

c
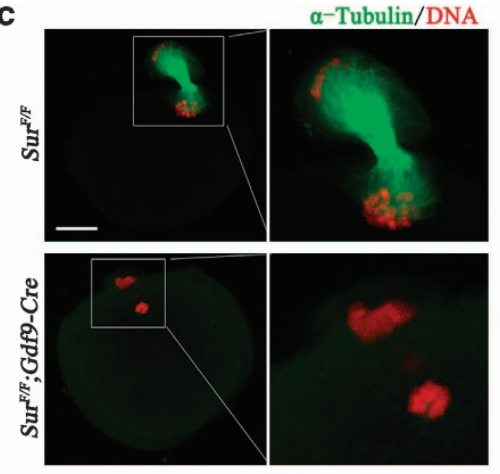

d

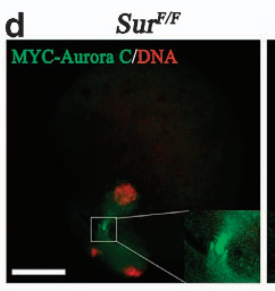

Sur ${ }^{k / F}$; Gdf9-Cre

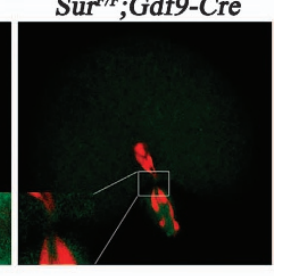

Figure 5 Cytokinesis failure in Sur ${ }^{F / F}$; Gdfg-Cre oocytes. (a and $\left.\mathbf{b}\right)$ Dynamics of F-actin during Sur ${ }^{F / F}$ and Sur ${ }^{F / F}$; Gdfg-Cre oocyte maturation. Oocytes were microinjected with Alexa 488-phalloidin and transferred to the live imaging station after $1 \mathrm{~h}$ of incubation. Time points indicated the time elapsed from the start of corresponding Supplementary Movie 3A and B. Red arrows showed actin ring in (a) and (b); white arrows indicated segregated chromosomes in (a) and (b); yellow arrows showed the polar body in (a) and membrane protrusion in (b). Bar $=25 \mu \mathrm{m}$. (c and d) Localization of midbody spindle and Myc-Aurora-C at telophase in Sur/F and Sur ${ }^{F / F}$; Gdf9-Cre oocytes. Oocytes used for staining were fixed after 8 h of incubation in the Sur ${ }^{F / F}$; Gdfg-Cre group and $12 \mathrm{~h}$ in the Sur ${ }^{F / F}$ group, respectively. Green: $\alpha$-tubulin in (c); Myc-Aurora C in (d); Red: DNA. Bar $=25 \mu \mathrm{m}$. (e) Scheme represents the process of polar body emission in oocytes of indicated genotypes

arrest induced by nocodazole. ${ }^{16}$ In the present study, indeed the absence of kinetochore localization of BubR1 and Bub3 in prometaphase I was observed in Survivindeleted oocytes. Furthermore, microinjection of the kinasedeficient Aurora C (AurC-KD) mRNA into mouse oocytes also disrupted Bub1 and BubR1 targeting to kinetochores and caused premature chromosome segregation. ${ }^{21}$ Interestingly, our data also showed that the kinetochore localization of Aurora C was disturbed in Survivin-depleted oocytes (data not shown).

Survivin is essential for the completion of cytokinesis in mouse oocyte meiotic maturation. In meiosis I, the course of PBE mainly includes three processes: the establishment of polarity, membrane protrusion and successful abscission. ${ }^{27,28}$ Survivin-deleted oocytes showed membrane protrusion but failed to extrude the polar body, suggesting that these defective oocytes completed the first two steps of PBE but failed to undergo the third step, that is, abscission. In Drosophila, Survivin facilitates the localization of Polo kinase and Rho at the equatorial cortex in spermatocytes, ${ }^{29}$ and these kinases may modify substrates like $\mathrm{CHO1/MKLP}$ that is responsible for assembly of the midbody microtubule complex. ${ }^{30}$ In human retinal pigment epithelial (RPE) cells, Survivin depletion disrupts the association of the inner centromere protein (INCENP),
Aurora-B and MKLP-1 with the spindle midzone and the midbody. ${ }^{25}$ In our study, we found that Survivin depletion disrupted the midbody microtubules in telophase I but did not affect the dynamic movement of F-actin to form a contractile ring. We also found that the kinase protein Aurora $\mathrm{C}$, which carries out functions in oocyte meiosis rather than Aurora $\mathrm{B},{ }^{31}$ was also disturbed in telophase in Survivin-depleted oocytes.

Survivin in GCs has fundamental roles in folliculogenesis. In a recent study, a known splice variant of Survivin, Survivin-2B, has been reported to regulate cell death. ${ }^{32}$ Here, our results showed that the Survivin depletion in GCs led to abnormal follicle growth, ovulation failure (follicular atresia) and impaired luteinization, which were caused by massive GCs apoptosis. In the absence of healthy GCs, ovarian follicles are destined to undergo atresia because of loss of the main supporting signals. It is known that $\mathrm{FSH}$ controls follicular development by regulating GC proliferation and differentiation; ${ }^{33,34}$ and $\mathrm{FSH}$ responsiveness is essential for the formation of the antrum and growth to the preovulatory stage. ${ }^{35}$ In our study, GC viability and differentiation to the preovulatory stage was impaired in the $S_{\text {Sur }}{ }^{F / F}$; Cyp19-Cre mice. The lower expression of the Cyp19a1 in the mutant GCs suggested a weakened FSH responsiveness. Then, the downregulation of the Fshr in mutant GCs 
a

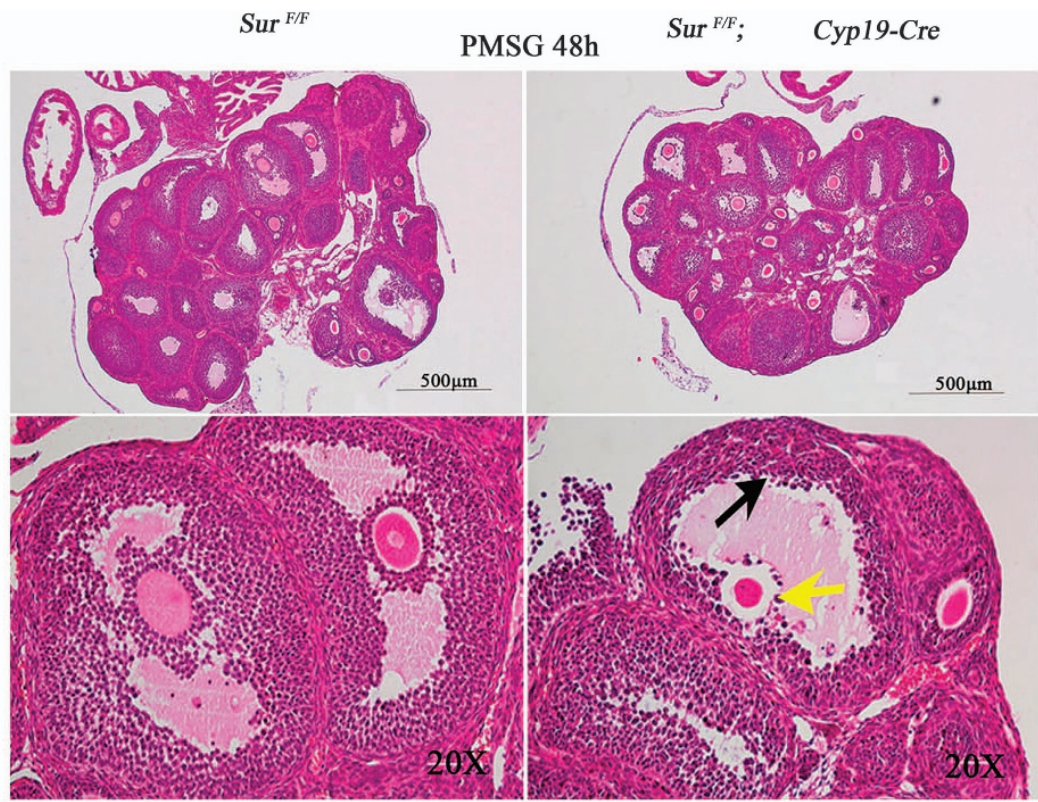

b

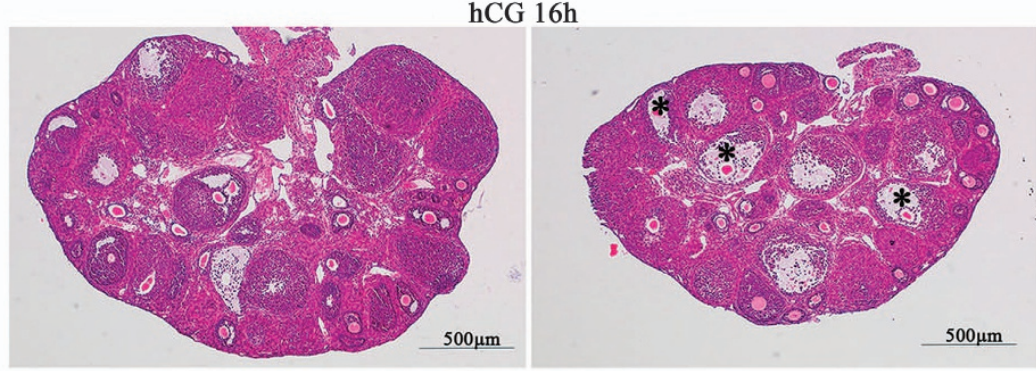

C

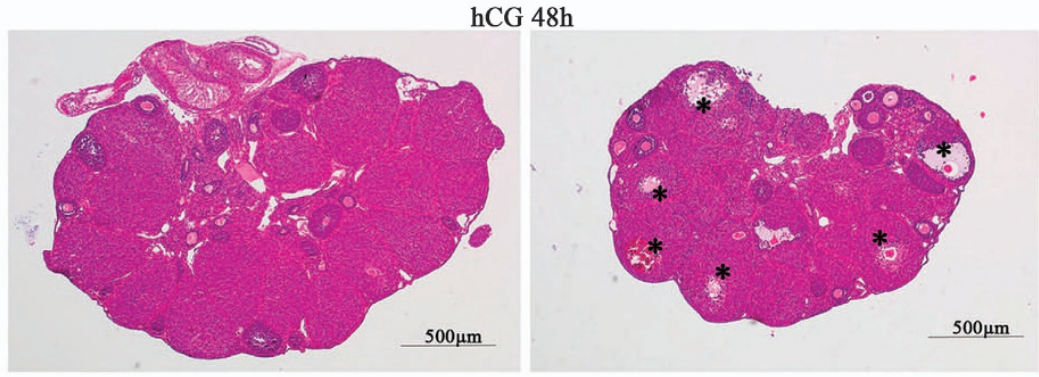

d
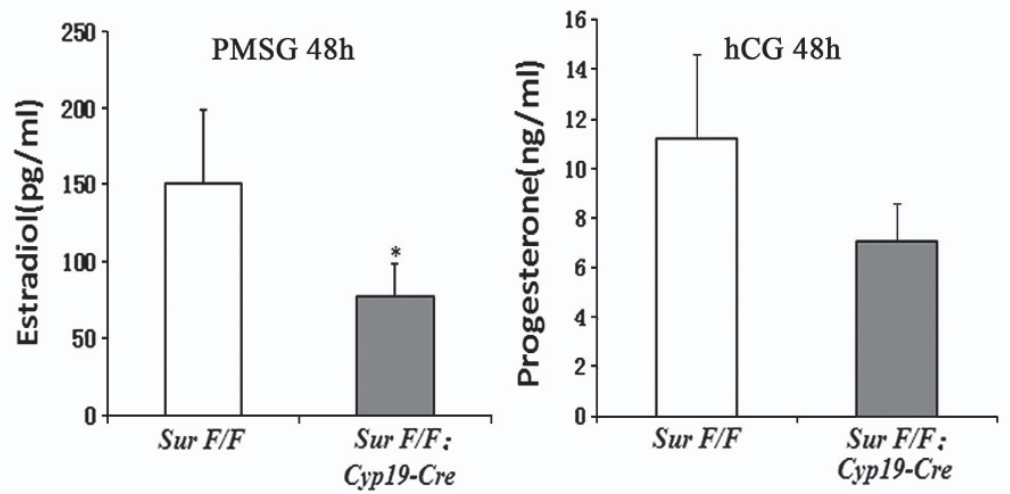

Figure 6 Impaired folliculogenesis, ovulation and $\mathrm{CL}$ development in Sur ${ }^{\mathrm{F} / F}$; Cyp19-Cre mice. (a) Representative H\&E staining of ovaries $48 \mathrm{~h}$ after PMSG priming of $\sim$ d23 female mice. Bar $=500 \mu \mathrm{m}$. The two panels below were at a $\times 20$ magnification (black arrow, the membrana GCs; yellow arrow, the cumulus cells). (b) Representative $\mathrm{H} \& \mathrm{E}$ staining of ovaries from mice primed with PMSG for $48 \mathrm{~h}$ at $\sim \mathrm{d} 23$, followed by $\mathrm{hCG}$ for $16 \mathrm{~h}$. Bar $=500 \mu \mathrm{m}$; asterisk, follicles that failed to ovulate $00 \mathrm{cytes}$. (c) Representative H\&E staining of ovaries from mice primed with PMSG for $48 \mathrm{~h}$ at $\sim \mathrm{d} 23$, followed by hCG for $48 \mathrm{~h}$. Bar $=500 \mu \mathrm{m}$, asterisk showed undeveloped CLs. (d) Levels of both serum estradiol and progesterone were decreased in Sur ${ }^{F / F}$; Cyp 19 -Cre mice. ${ }^{*} P<0.05$ 
a

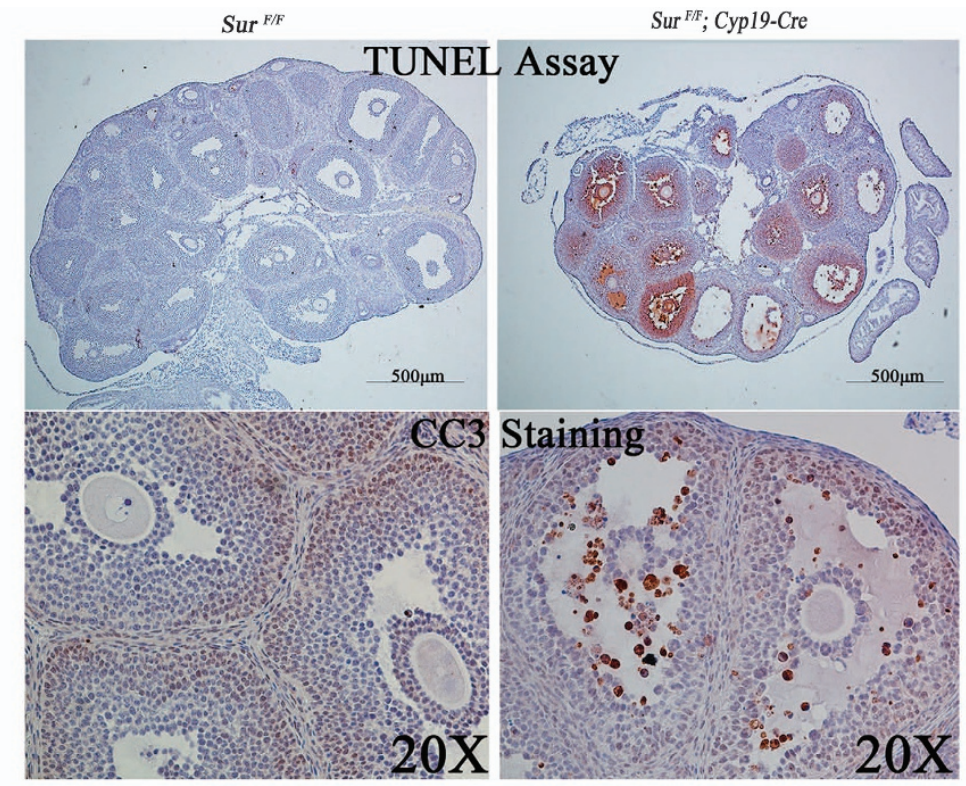

C
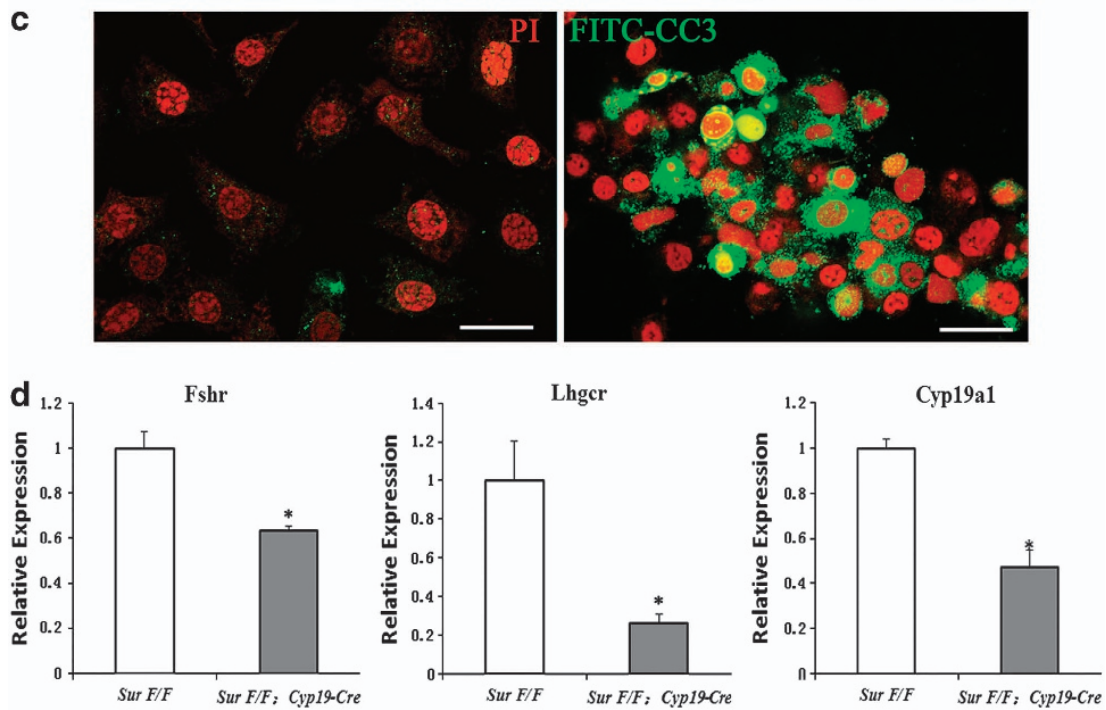

Figure 7 Survivin deletion led to GC apoptosis and downregulation of key ovarian genes. (a and $\mathbf{b}$ ) TUNEL assay and immunostaining of CC3 in ovaries from control and Sur ${ }^{F / F}$; Cyp19-Cre mice treated with PMSG for $48 \mathrm{~h}$ showing GC apoptosis. (c) Immunofluorescence for CC3 in cultured GCs showed more apoptotic cells in Survivin-depleted GCs. Green, CC3; Red, DNA. Bar $=20 \mu \mathrm{m}$. (d) Relative expression of key ovarian genes (Fshr, Lhgcr and Cyp19a1) in control and Sur ${ }^{F / F}$; Cyp19-Cre mice after PMSG treatment. ${ }^{*} P<0.05$

provided a molecular mechanism that might explain the poor follicle growth in Sur ${ }^{/ / F}$; Cyp19-Cre mice. On the other hand, $\mathrm{LH}$ initiates ovulation, terminates GC proliferation and regulates the transition of GCs to lutea cells (LCs). ${ }^{36}$ Here, we demonstrated a dramatic decrease of Lhgcr expression in the mutant GCs, which might account for the reduced ovulation and impaired $\mathrm{CL}$ formation observed in $\mathrm{Sur}^{\mathrm{F} / \mathrm{F}}$; Cyp19-Cre mice.

A recent report showed that human oocytes/embryos not only expressed but also secreted surviving, suggesting that Survivin may have an important role in human oogenesis and embryogenesis. ${ }^{37}$ Thus, our results may provide new insights for understanding functions of Survivin in human oogenesis, which will aid in understanding pathology of human infertility or subfertility.

\section{Materials and Methods}

Mice. The Survivin floxfllox (referred to as Sur/F) mice in a C57BL/6J genomic background were provided by Tak W Mak. ${ }^{15}$ Transgenic mouse lines carrying growth differentiation factor 9 (Gdf-9) and cytochrome P450 (Cyp-19) promotermediated Cre recombinase were provided by Dr. Heng-Yu Fan. ${ }^{38,39}$ Both transgenic mouse lines have C57BL/6J genomic background. The Sur ${ }^{F / F}$ mice were crossed with Gdf9-Cre mice and Cyp19-Cre mice, respectively. After multiple rounds of crossing, we had generated mutant female mice lacking Survivin in oocytes (referred to as Sur ${ }^{F / F}$; Gdfg-Cre) and GCs (referred to as Sur ${ }^{F / F}$; Cyp19$\mathrm{Cre}$ ). The mice were housed under controlled environmental conditions with free access to water and food. Illumination was provided between 0800 and $2000 \mathrm{~h}$.

Oocyte collection and culture. Animal care and handling were conducted according to the guidelines of the Animal Research Committee of the Institute of Zoology, Chinese Academy of Sciences. GV stage oocytes were isolated from ovaries of 6- to 9-week-old female mice and cultured in M2 medium (Sigma, St. Louis, MO, USA) under paraffin oil at $37^{\circ} \mathrm{C}, 5 \% \mathrm{CO}_{2}$ in air. They were collected 
at different times of culture for immunofluorescent staining, western blotting, microinjection and chromosome spreads.

Superovulation. To induce synchronized follicular growth and ovulation, each female mouse at $\sim$ d23 was injected with $7.5 \mathrm{IU}$ of PMSG followed by $7.5 \mathrm{IU}$ of hCG $48 \mathrm{~h}$ to promote ovulation. Mice were killed at $12-14 \mathrm{~h}$ or $16 \mathrm{~h}$ of $\mathrm{hCG}$ treatment and COCs were recovered from each oviduct. After a 5-min treatment with hyaluronidase $(1 \mathrm{mg} / \mathrm{ml})$ in M2 medium (Sigma), oocytes were collected and counted.

Antibodies, immunofluorescence and confocal microscopy. Antibodies used in the experiments were purchased from the following companies: rabbit monoclonal anti-Survivin (Cell Signaling Technology, Beverly, MA, USA); mouse monoclonal anti- $\alpha$-tubulin, anti- $\gamma$-tubulin (Sigma), anti-myc-FITC, anti-GAPDH (Invitrogen, Carlsbad, CA, USA); sheep polyclonal anti-BubR1 (Abcam, Cambridge, MA, USA); rabbit polyclonal anti-Bub3 (Santa Cruz, Dallas, TX, USA). Secondary antibodies were purchased from ZhongShan Golden Bridge Biotechnology Co., Ltd (Beijing, China). Oocytes for immunofluorescent staining were fixed in $4 \%$ paraformaldehyde in PBS for $30 \mathrm{~min}$ at room temperature. Then, they were transferred to membrane permeabilization solution $(0.5 \%$ Triton X-100) for $20 \mathrm{~min}$ and blocking buffer (1\% BSA-supplemented PBS) for $1 \mathrm{~h}$. At last, oocytes were incubated overnight at $4{ }^{\circ} \mathrm{C}$ with antibodies described above in appropriate dilutions. Then, the oocytes were mounted on glass slides and examined with a laser scanning confocal microscope (Zeiss LSM 710 META, Oberkochen, Germany).

cDNA cloning, mRNA synthesis and microinjection. Mouse Aurora C cDNA was obtained by RT-PCR using total RNA from 150 mouse oocytes. The following two nested primers were used: F1: ACTTCAACTTGCCCTCAC, R1: ACAGAGCCTGGAGACCTT; F2: TCAGGCCGGCCGATGGAGCCCAGCACCTCA ACCA, R2: GTTGGCGCGCCCTAGCAAGGAAGCACCCTTCGA. The full Aurora C cDNA was then cloned to pSC2 + -Myc vector. The Myc-Aurora C-pCS2 + plasmid was linearized by Sall and purified by the gel extract kit (Promega, Madison, WI, USA). SP6 message machine (Ambion, Austin, TX, USA) was used for producing capped mRNA which was then purified by the RNeasy cleanup kit (Qiagen, Hilden, Germany). Microinjection was performed using an Eppendorf microinjector (Hamburg, Germany) and completed within $30 \mathrm{~min}$.

Live imaging of oocytes and chromosome spread. For live oocyte imaging, oocytes were respectively microinjected with $\beta 5$-tubulin-GFP mRNAs, Cyclin B1-GFP mRNAs or Alexa 488-phalloidin (Invitrogen). The oocytes were cultured in M2 medium with Hoechst $33342(5 \mathrm{ng} / \mathrm{ml})$ for $2 \mathrm{~h}$ and then transferred to the Perkin Elmer precisely Ultra VIEW VOX Confocal Imaging System (PerkinElmer, Waltham, MA, USA) equipped with $37^{\circ} \mathrm{C}$ incubator and $5 \% \mathrm{CO}_{2}$ supply. The DNA in oocytes was labeled in blue using Hoechst 33342 , and changed into red in the figures and movies.

For chromosome spreads, oocytes were left in hypotonic solution ( $1 \% \mathrm{Na}$ Citrate) for $20 \mathrm{~min}$ at room temperature and fixed with methanol/glacial acetic acid $(3: 1)$. The oocytes were then stained with PI for $5 \mathrm{~min}$.

Histological analysis, immunohistochemistry and TUNEL assay. Ovaries used for histological analysis were collected from female mice after a PMSG 48-h treatment. Then, they were fixed in $4 \%$ paraformaldehyde $(\mathrm{pH}$ 7.5) overnight at $4^{\circ} \mathrm{C}$, dehydrated, and embedded in paraffin. Paraffin-embedded ovaries were sectioned at a thickness of $8 \mu \mathrm{m}$ for hematoxylin and eosin (H\&E) staining. One or both ovaries from more than three mice of each genotype were used for each time point.

Immunohistochemistry was performed on $8-\mu \mathrm{m}$ sections using the Vectastain ABC kit (Vector Laboratories, Burlingame, CA, USA). Rabbit monoclonal antiSurvivin was used to detect the expression of Survivin in mouse ovary. Rabbit anti-phosphohistone H3 (Upstate Laboratories, Lake Placid, NY, USA) and rabbit anti-cleaved caspase-3 (Cell Signaling Technology) were used to evaluate cell proliferation and apoptosis in follicles. For direct comparison, ovary sections from four individual females of each genotype were processed together.

Analysis of apoptosis in ovarian follicles was carried out by TUNEL assay using the ApopTag Plus in situ apoptosis detection kit (Chemicon International, Temecula, CA, USA). At least four different specimens from each genotype were analyzed in parallel.

GCs isolation, culture and immunofluorescence. Immature female mice ( $\sim$ d23) were injected with 7.5 IU PMSG, and ovaries were harvested $48 \mathrm{~h}$ later. Ovaries were dissected and placed in DMEM/F12. Large antral follicles were punctured to extrude GCs, which were then strained and spun down. GCs were harvested and cultured in DMEM/ F12 medium on cover glasses in culture dishes. After $24 \mathrm{~h}$ of culture, cells were fixed in $4 \%$ paraformaldehyde and immunofluorescent staining was performed as previously described ${ }^{40}$ with primary anti-cleaved caspase-3 antibody and secondary FITC-conjugated goat anti-rabbit IgG antibody (ZhongShan Golden Bridge Biotechnology Co.).

Western blot analysis. A total of 250 mouse oocytes per sample or GC extracts containing $30 \mu \mathrm{g}$ protein were mixed with SDS sample buffer and boiled for $5 \mathrm{~min}$ at $100^{\circ} \mathrm{C}$ for SDS-PAGE. Western blotting was performed as described previously ${ }^{40}$ using the antibody dilution anti-Survivin: 1:1000; anti-GAPDH: 1:2000.

RNA isolation and quantitative real-time PCR analysis. Total RNA from primary mouse GCs was extracted using the RNeasy micro purification kit (Qiagen). Single-stranded cDNAs were generated with the cDNA synthesis kit (Invitrogen). The resultant cDNAs were used for real-time PCR. Real-time PCR was conducted by using SYBR Premix Ex Taq kit (TaKaRa Biotechnology (Dalian) Co., Ltd., Japan) in ABI prism 7500 Sequence Detection System (Applied Biosystems, Foster City, CA, USA). Analysis of relative gene expression was measured by real-time quantitative PCR and the $2^{-\Delta \Delta C T}$ method.

Serum analysis. Mice were anesthetized after PMSG $48 \mathrm{~h}$ or PMSG 48 $\mathrm{h}$-hCG $48 \mathrm{~h}$ treatment and blood was collected by eyeball removal. Estradiol and progesterone measurements were made at a commercial laboratory (Chemclin Co., Beijing, China). At least 10 mice were anesthetized for each group.

Statistical analysis. All experiments were repeated at least three times. Statistical analysis was performed using SPSS (SPSS China, Shanghai, China). Data were expressed as mean \pm S.E.M. and $P<0.05$ was considered as statistically significant.

\section{Conflict of Interest}

The authors declare no conflict of interest.

Acknowledgements. We thank Hua Qin, Yi Hou, Shi-Wen Li and Li-Juan Wang for their technical assistance. We also thank Jun-Yu Ma for providing the plasmids Cyclin B1-GFP. This work was supported by the National Basic Research Program of China (2012CB944404, 2011CB944501) and the National Natural Science Foundation of China $(31272260,31371451)$.

1. Oktem O, Urman B. Understanding follicle growth in vivo. Hum Reprod 2010; 25: 2944-2954.

2. Sun QY, Miao YL, Schatten H. Towards a new understanding on the regulation of mammalian oocyte meiosis resumption. Cell Cycle 2009; 8: 2741-2747.

3. Yin S, Sun XF, Schatten $H$, Sun QY. Molecular insights into mechanisms regulating faithful chromosome separation in female meiosis. Cell Cycle 2008; 7: 2997-3005.

4. Matsuda F, Inoue N, Manabe N, Ohkura S. Follicular growth and atresia in mammalian ovaries: regulation by survival and death of granulosa cells. J Reprod Dev2012; 58: 44-50.

5. Tilly JL, Kowalski KI, Johnson AL, Hsueh AJ. Involvement of apoptosis in ovarian follicular atresia and postovulatory regression. Endocrinology 1991; 129: 2799-2801.

6. Inoue N, Matsuda F, Goto Y, Manabe N. Role of cell-death ligand-receptor system of granulosa cells in selective follicular atresia in porcine ovary. J Reprod and Dev 2011; 57 : 169-175.

7. Ambrosini G, Adida C, Altieri DC. A novel anti-apoptosis gene, survivin, expressed in cancer and lymphoma. Nat Med 1997; 3: 917-921.

8. Marusawa H, Matsuzawa S, Welsh K, Zou H, Armstrong R, Tamm I et al. HBXIP functions as a cofactor of survivin in apoptosis suppression. EMBO J 2003; 22: 2729-2740.

9. Kanwar JR, Kamalapuram SK, Kanwar RK. Targeting survivin in cancer: the cell-signalling perspective. Drug Discov Today 2011; 16: 485-494.

10. Jeyaprakash AA, Klein UR, Lindner D, Ebert J, Nigg EA, Conti E. Structure of a SurvivinBorealin-INCENP core complex reveals how chromosomal passengers travel together. Cell 2007; 131: 271-285.

11. Vader G, Kauw JJ, Medema RH, Lens SM. Survivin mediates targeting of the chromosomal passenger complex to the centromere and midbody. EMBO Rep 2006; 7: 85-92.

12. Uren AG, Wong L, Pakusch M, Fowler KJ, Burrows FJ, Vaux DL et al. Survivin and the inner centromere protein INCENP show similar cell-cycle localization and gene knockout phenotype. Curr Biol 2000; 10: 1319-1328. 
13. Delvaeye M, De Vriese A, Zwerts F, Betz I, Moons M, Autiero M et al. Role of the zebrafish survivin genes in vasculo-angiogenesis, neurogenesis, cardiogenesis and hematopoiesis. BMC Dev Biol 2009; 9: 25.

14. Jiang $\mathrm{Y}$, de Bruin A, Caldas H, Fangusaro J, Hayes J, Conway EM et al. Essential role for survivin in early brain development. J Neurosci 2005; 25: 6962-6970.

15. Okada H, Bakal C, Shahinian A, Elia A, Wakeham A, Suh WK et al. Survivin loss in thymocytes triggers p53-mediated growth arrest and p53-independent cell death. $J$ Exp Med 2004; 199: 399-410.

16. Li M, Li S, Yuan J, Wang ZB, Sun SC, Schatten H et al. Bub3 is a spindle assembly checkpoint protein regulating chromosome segregation during mouse oocyte meiosis. PLoS One 2009; 4: e7701.

17. Wei L, Liang XW, Zhang QH, Li M, Yuan J, Li S et al. BubR1 is a spindle assembly checkpoint protein regulating meiotic cell cycle progression of mouse oocyte. Cell Cycle 2010; 9: 1112-1121.

18. Sudakin V, Chan GK, Yen TJ. Checkpoint inhibition of the APC/C in HeLa cells is mediated by a complex of BUBR1, BUB3, CDC20, and MAD2. J Cell Biol 2001; 154: 925-936.

19. Homer H, Gui L, Carroll J. A spindle assembly checkpoint protein functions in prophase arrest and prometaphase progression. Science 2009; 326: 991-994

20. Schindler K, Davydenko O, Fram B, Lampson MA, Schultz RM. Maternally recruited Aurora C kinase is more stable than Aurora B to support mouse oocyte maturation and early development. Proc Natl Acad Sci USA 2012; 109: E2215-E2222.

21. Yang KT, Li SK, Chang CC, Tang CJ, Lin YN, Lee SC et al. Aurora-C kinase deficiency causes cytokinesis failure in meiosis I and production of large polyploid oocytes in mice. Mol Biol Cell 2010; 21: 2371-2383.

22. Carlton JG, Caballe A, Agromayor M, Kloc M, Martin-Serrano J. ESCRT-III governs the Aurora B-mediated abscission checkpoint through CHMP4C. Science 2012; 336: 220-225.

23. Lens SM, Wolthuis RM, Klompmaker R, Kauw J, Agami R, Brummelkamp T et al. Survivin is required for a sustained spindle checkpoint arrest in response to lack of tension. EMBO J 2003; 22: 2934-2947.

24. Carvalho A, Carmena M, Sambade C, Earnshaw WC, Wheatley SP. Survivin is required for stable checkpoint activation in taxol-treated HeLa cells. J Cell Sci 2003; 116(Pt 14): 2987-2998.

25. Yang D, Welm A, Bishop JM. Cell division and cell survival in the absence of survivin Proc Natl Acad Sci USA 2004; 101: 15100-15105.

26. Sun SC, Wei L, Li M, Lin SL, Xu BZ, Liang XW et al. Perturbation of survivin expression affects chromosome alignment and spindle checkpoint in mouse oocyte meiotic maturation. Cell Cycle 2009; 8: 3365-3372.

27. Azoury J, Lee KW, Georget V, Hikal P, Verlhac MH. Symmetry breaking in mouse oocytes requires transient F-actin meshwork destabilization. Development 2011; 138: 2903-2908.

28. Dehapiot B, Carriere V, Carroll J, Halet G. Polarized Cdc42 activation promotes polar body protrusion and asymmetric division in mouse oocytes. Dev Biol 2013; 377: 202-212.

29. Szafer-Glusman E, Fuller MT, Giansanti MG. Role of Survivin in cytokinesis revealed by a separation-of-function allele. Mol Biol Cell 2011; 22: 3779-3790.
30. Straight AF, Field CM. Microtubules, membranes and cytokinesis. Curr Biol. 2000; 10: R760-R770.

31. Avo Santos M, van de Werken C, de Vries M, Jahr H, Vromans MJ, Laven JS et al. A role for Aurora $\mathrm{C}$ in the chromosomal passenger complex during human preimplantation embryo development. Hum Reprod 2011; 26: 1868-1881.

32. Shi K, An J, Shan L, Jiang Q, Li F, Ci Y et al. Survivin-2B promotes autophagy by accumulating IKK alpha in the nucleus of selenite-treated NB4 cells. Cell Death Dis 2014; 5: e1071.

33. Rao MC, Midgley Jr AR, Richards JS. Hormonal regulation of ovarian cellular proliferation. Cell 1978; 14: 71-78

34. Richards JS, Fitzpatrick SL, Clemens JW, Morris JK, Alliston T, Sirois J. Ovarian cell differentiation: a cascade of multiple hormones, cellular signals, and regulated genes. Recent Prog Horm Res 1995; 50: 223-254.

35. Richards JS, Russell DL, Ochsner S, Hsieh M, Doyle KH, Falender AE et al. Nove signaling pathways that control ovarian follicular development, ovulation, and luteinization. Recent Prog Horm Res 2002; 57: 195-220.

36. Robker RL, Richards JS. Hormonal control of the cell cycle in ovarian cells: proliferation versus differentiation. Biol Reprod 1998; 59: 476-482.

37. Balakier H, Xiao R, Zhao J, Zaver S, Dziak E, Szczepanska K et al. Expression of survivin in human oocytes and preimplantation embryos. Fertil Steril 2013; 99: 518-525.

38. Lan ZJ, Xu X, Cooney AJ. Differential oocyte-specific expression of Cre recombinase activity in GDF-9-iCre, Zp3cre, and Msx2Cre transgenic mice. Biol Reprod 2004; 71: 1469-1474.

39. Fan HY, Liu Z, Shimada M, Sterneck E, Johnson PF, Hedrick SM et al. MAPK3/1 (ERK1/2) in ovarian granulosa cells are essential for female fertility. Science 2009; 324: 938-941.

40. Zhang D, Ma W, Li YH, Hou Y, Li SW, Meng XQ et al. Intra-oocyte localization of MAD2 and its relationship with kinetochores, microtubules, and chromosomes in rat oocytes during meiosis. Biol Reprod 2004; 71: 740-748.

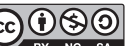

Cell Death and Disease is an open-access journal published by Nature Publishing Group. This work is licensed under a Creative Commons Attribution-NonCommercialShareAlike 3.0 Unported License. The images or other third party material in this article are included in the article's Creative Commons license, unless indicated otherwise in the credit line; if the material is not included under the Creative Commons license, users will need to obtain permission from the license holder to reproduce the material. To view a copy of this license, visit http://creativecommons.org/licenses/ by-nc-sa/3.0/

Supplementary Information accompanies this paper on Cell Death and Disease website (http://www.nature.com/cddis) 